\title{
Logic and Cognition: Special Issue of Best Papers of the ESSLLI 2012 Workshop
}

\author{
Jakub Szymanik • Rineke Verbrugge
}

Published online: 11 January 2014

(C) Springer Science+Business Media Dordrecht 2014

The explanatory power of logic is vast and therefore it has proved a valuable tool for many disciplines, including the building-blocks of cognitive science, such as philosophy, computer science, mathematics, artificial intelligence, and linguistics. Logic has a great track record in providing interesting insights by means of formalization, and as such it is very useful in disambiguating psychological theories. Logically formalized cognitive theories are not only the source of unequivocal experimental hypotheses, but they also lend themselves naturally to computational modeling. Most importantly, modern logic has at its service a rich variety of tools to assess and compare such psychological theories. This toolbox can be utilized in evaluating cognitive models along the following dimensions:

(a) logical relationships, for example, incompatibility or identity of models;

(b) explanatory power, for example, what can be expressed by means of a model?

(c) computational plausibility, for example, are computations postulated by cognitive models tractable, do they scale-up?

In this way, logic can play a major role in the process of psychological explanation. For some interesting examples, see Stenning and van Lambalgen (2008).

Marr (1983) has argued that any particular task computed by a cognitive system must ultimately be analyzed at three levels of explanation (in order of decreasing abstraction):

\footnotetext{
J. Szymanik $(\varangle)$

Institute for Logic, Language, and Computation, University of Amsterdam,

P. O. Box 94242, 1098 XG, Amsterdam, The Netherlands

e-mail: J.K.Szymanik@uva.nl

R. Verbrugge

Institute of Artificial Intelligence, University of Groningen,

P. O. Box 407, 9700 AK, Groningen, The Netherlands

e-mail: 1.c.verbrugge@rug.nl
} 
1. the computational level (the problem solved or function computed);

2. the algorithmic level (the algorithm used to achieve a solution); and

3. the implementation level (how the algorithm is actually implemented in neural activity).

As we have argued elsewhere (Isaac et al. 2014), logic has much to offer on every level but can also help extending the levels of explanations. For instance, using the descriptive power of logic we can study the territory between the computational level and the algorithmic level. Given a computational explanation of a particular cognitive task, we can logically study its intrinsic properties (for example, its computational complexity). As a result of such logical analysis we can a priori restrict the class of possible algorithmic implementations of the cognitive problem, thereby producing a narrower class of experimental hypotheses. In a slogan, we can go from logic to human behaviour.

The last few decades, one of the dominating programs in cognitive science has been to use Bayesian inference, following Anderson's strategy of Rational Analysis (Anderson 1991). The Principle of Rationality asserts that the most powerful explanation of human cognition is to be found via analysis at the computational level, under the assumption that task performance has been optimized rather on an evolutionary timescale than via analysis of underlying mechanisms. In his famous 1957 critique of the model of people as fully rational, Simon had already proposed the Principle of Bounded Rationality, which also takes into account goals and limited resources of the agents (Simon 1957). Logical analysis of a computational task is usually necessary to discover the parameters that make a particular cognitive challenge easy or hard for bounded agents (see, e.g., Szymanik et al. 2013), and therefore studying the logical/computational core of a cognitive task can help to resolve the tension between Rational Analysis and more mechanistic approaches.

The actual interactions between psychologists and logicians have, however, been very rare until fairly recently. This situation is even more puzzling as we see a growing number of cognitive science groups concerned with powerful mathematical and computational techniques, such as Bayesian statistics (Tenenbaum et al. 2011). Even though, as we have explained above, logic can help to bridge the gap between various types of cognitive models, there has so far been no single psychological lab focused around logic itself. We think that in the long run, it will be necessary for the success of cognitive science to adopt some of the unifying perspective that logic can provide.

Right now, it seems that there are no pressing scientific issues delaying the successful marriage of two disciplines; the main hindrance seems to be a deep sociological divide between two fields. We believe that the best way of breaking the impasse is by displaying examples of mutually fruitful and beneficial collaboration. In this special issue we gathered six papers doing just that. When we compare this special issue to previous special issues on logic and cognition, ${ }^{1}$ it is striking that, whereas the earlier issues contained quite a few programmatic and theoretical papers, all current arti-

\footnotetext{
1 See the 2007 special issue of Topoi on "Logic and Cognition", ed. van Benthem, Hodges, and Hodges; the 2008 special issue of Journal of Logic, Language and Information on "Formal Models for Real People", ed. Counihan and van Lambalgen; the 2008 special issue of Studia Logica on "Psychologism in Logic?", ed. Leitgeb.
} 
cles are either based on the authors' own experimental work or make predictions for concrete experiments. Logic has really entered the lab! Let us give a brief overview.

\section{Cognition and Formal Models of Natural Language Use}

Sentences like "All students followed each other into the room" and "All students know each other" are notoriously difficult to interpret. The first sentence appears to call for a path interpretation, in which a path consisting of all students walks into the room. The second sentence calls up the idea of a complete graph, in which each pair of students knows one another. Schlotterbeck and Bott provide interesting formal and empirical analyses of such sentences in their article "Easy solutions for a hard problem? The computational complexity of reciprocals with quantificational antecedents". The authors have been inspired, amongst other work, by Iris van Rooij's "Tractable Cognition Thesis" (van Rooij 2008) and by Szymanik's earlier research on the complexity of model checking for various kinds of quantified sentences in given finite models (Szymanik 2009, 2010).

Before performing their experiments, they assume as a possibility that, based on previous experience, people who have to interpret a sentence like "Exactly five dots are directly connected with each other" stay away from interpretations that in the past were too complex for them to verify. This avoidance strategy turns out to more or less correspond with the data that Schlotterbeck and Bott find in their experiments. However, they also find that people sometimes do arrive at computationally intractable interpretations, and then may try to solve the problem of verifying the sentence in a picture by guessing and pattern recognition. The authors cautiously conclude that restricting meanings to those verifiable in polynomial time might be too too strict and suggest looking at broader notions of complexity, e.g., meanings verifiable by non-deterministic Turing machines (Ristad 1993; Mostowski and Szymanik 2012) or fixed-parameter tractability (van Rooij 2008).

In her contribution "Linguistic and visual cognition: Verifying proportional and superlative most in Bulgarian and Polish", Barbara Tomaszewicz continues the theme of the complexity of interpreting sentences with generalised quantifiers. The author concentrates her investigation on a phenomenon that occurs in languages like Bulgarian and Polish, but not in English, namely that they have two different words that correspond to two different meanings of most. Let us call the expression with usual majority interpretation most ; then Polish and Bulgarian also have a quantifier for most2, where "Most 2 Dutch people vote for Geert Wilders" means "The Wildersvoters form the largest subset"; this might be true in situations where "Most1 Dutch people vote for Geert Wilders" is false.

This phenomenon provides a fruitful background for investigating which algorithms people might use when checking whether sentences like "Mostl dots are yellow" or "Most 2 dots are yellow" are true for a certain picture with dots of several colours and sentences. Does people's model checking strategy depend mostly on sentence form or do people rely on properties of the picture, such as whether it contains dots of only two colours or more? These questions follow up on Lidz's Interface Transparency Thesis (Lidz et al. 2011), which states that "the verification procedures employed 
in understanding a declarative sentence are biased towards algorithms that directly compute the relations and operations expressed by the semantic representation of that sentence" - and for the English "Most dots are yellow", Lidz had argued on the basis of experiments with English-speaking subjects that people would most likely approximate the number of yellow dots and check whether it is larger than the number of all dots minus that of yellow dots. Tomaszewicz' experiments with Bulgarianand Polish-speaking subjects replicated Lidz's findings as far as most 1 is concerned, whereas most 2 is verified by stepwise selection: "there are more yellow dots than red ones and there are more yellow dots than blue ones and ..."; and that participants' behaviour is triggered by the semantics of the two quantifiers, much more than by the properties of the picture, even if the latter could have delivered a more efficient strategy.

The paper by Krzyżanowska, Wenmackers and Douven, "Inferential conditionals and evidentiality", investigates the phenomenon that people would find the use of a conditional "If $p$, then $q$ " felicitous only if there is some kind of inferential link between the antecedent and the consequent. The authors base their analysis on Douven's and Verbrugge's typology (Douven and Verbrugge 2010): $q$ may be either a deductive consequence, an inductive consequence, or an abductive consequence of $p$; and in all three cases, the inference may depend not only on $p$ but also on contextually given background assumptions. In their first experiment, the authors check of several conditionals whether they are considered to be more or less assertable when expressions like "must", "should" or "probably" are inserted in the consequent. In English, it turned out that in general, such markers lowered the assertability of the conditionals. However, when looking per inference type, there could be a positive influence, such as the marker "should", which appeared to serve as an inductive inferential marker; the marker "must", which had a positive effect in adductive inferences; and "probably", which did very well with conditionals that have abductive and inductive consequence relations. In contrast, a marker such as "probably" would decrease the assertability of conditionals with deductive consequence relations. The results were similar for Dutch.

\section{Logical Models of Reasoning}

Égré, de Gardelle and Ripley, in their article "Vagueness and Order Effects in Color Categorization", present experimental results that are meant to adjudicate between several pre-existing hypotheses from the literature on vague predicates. How do people evaluate borderline cases of predicates like blue and green? Is there underlap, in the sense that there is a gap between the extension of the positive and negative extension of blue? Or is there overlap, in the sense that colours on the border between blue and green may be called "blue and not blue" or "blue and green"? Or is there simply a sharp boundary between blue and not blue, only we are uncertain of its exact location?

By using a series of colour shades, for example from green to blue, in one part of an experiment, the authors asked subjects to evaluate the shades "perceptually": Given a blue and a green comparison square, is a newly presented colour square more similar to the green or the blue one? In a second part of the experiment, the subjects had to do a corresponding "linguistic" task: Is the newly presented square blue or green? 
The authors find a phenomenon of negative hysteresis for the linguistic version, which means that the subjects switch earlier from the evaluation green to blue when they move though a gradual transition of colour shades from green to blue, than when the order is reversed. In the perceptual task, a critical boundary was found-subjects switched categories at a relatively stable position, in whichever order the coloured squares were presented to them. The outcomes of this experiment and a follow-up defeats the authors' initial expectation of hysteresis, based on the argument that in the Sorites paradox, one is tempted to stay with the category one is coming from.

Hamami and Mumma investigate the use of diagrams in Euclid's Elements in their contribution "Prolegomena to a cognitive investigation of Euclidean diagrammatic reasoning". They aim to confront a logical and philosophical analysis of diagrammatic reasoning in geometrical proofs with insights from cognitive science, specifically, mathematical cognition, spatial cognition, and the psychology of reasoning. They develop interesting analyses of cognitive representations and processes in Euclidean diagrammatic reasoning, also paying attention to the complexity of this special type of reasoning. Even though the authors have not yet done experiments in the lab themselves to validate their hypotheses, on the basis of their analyses they do sketch some proposals for fascinating new experiments to be done in future.

Finally, Gierasimczuk, van der Maas and Raijmakers in their article "An analytic model for Deductive Mastermind empirically tested with a massively used online learning system" fruitfully apply a specific form of logical proofs, namely analytic tableaux, to investigate the difficulty of different game items as experienced by children. Deductive Mastermind is a simplification of the usual Mastermind in that the player only needs to come up with the right answer for the opponent's secret pattern of flowers based on clues and feedback in terms of coloured pins standing for the three feedback types "wrong colour", "right colour, wrong place", and "right colour, right place". It turns out that the size of the analytical tableaux that one would make for an item corresponds very nicely with the difficulty of game items as experienced by the children. Also, error types and their frequency correspond with tableau representation. It appears that the analytic tableau analysis is a very useful tool to assess complexity of reasoning tasks, and may in future also be applied to, for example, Sudoku.

Acknowledgments We would like to thank all the people who helped to bring about this special issue. First of all, we thank all speakers at the ESSLLI 2012 workshop on Logic and Cognition for ensuring a diverse and interesting workshop. Special thanks are due to the members of the program committee of the workshop for their dedication to provide authors with useful, constructive feedback: Leon de Bruin, Eve Clark, Robin Clark, Paul Égré, Fritz Hamm, Alice ter Meulen, Marcin Mostowski, Maartje Raijmakers, Iris van Rooij, Keith Stenning, and Marcin Zajenkowski. All workshop papers have been published in Szymanik and Verbrugge (2012). We would also like to express our gratitude to the anonymous reviewers of this special issue, who have written constructive reviews with many detailed suggestions for the authors. Finally we would like to thank all authors who submitted their work to this issue for showing patience in dealing not only with the wishes of reviewers, but also with two quite demanding editors.

\section{References}

Anderson, J. R. (1991). The place of cognitive architectures in a rational analysis. In K. Van Lehn (Ed.), Architectures for intelligence (pp. 1-21). Hillsdale, NJ: Lawrence Erlbaum Associates.

Douven, I., \& Verbrugge, S. (2010). The Adam's family. Cognition, 117(3), 302-318. 
Isaac, A., Szymanik, J., \& Verbrugge, R. (2014). Logic and complexity in cognitive science, Outstanding Contributions. In A. Baltag \& S. Smets (Eds.), Johan van Benthem on logical and informational dynamics, Trends in Logic. Berlin: Springer.

Lidz, J., Pietroski, P., Halberda, J., \& Hunter, T. (2011). Interface transparency and the psychosemantics of most. Natural Language Semantics, 19(3), 227-256.

Marr, D. (1983). Vision: A computational investigation into the human representation and processing visual information. San Francisco, CA: W.H. Freeman.

Mostowski, M., \& Szymanik, J. (2012). Semantic bounds for everyday language. Semiotica, 188(1-4), 363-372.

Ristad, E. S. (1993). The language complexity game. Artificial intelligence. Cambridge, MA: MIT Press.

Simon, H. A. (1957). Models of man: Social and rational. New York, NY: Wiley.

Stenning, K., \& van Lambalgen, M. (2008). Human reasoning and cognitive science. Cambridge, MA: MIT Press.

Szymanik, J. (2009). Quantifiers in TIME and SPACE. Computational complexity of generalized quantifiers in natural language. $\mathrm{PhD}$ thesis. Amsterdam: University of Amsterdam.

Szymanik, J. (2010). Computational complexity of polyadic lifts of generalized quantifiers in natural language. Linguistics and Philosophy, 33(3), 215-250.

Szymanik, J., Meijering, B., \& Verbrugge, R. (2013) Using intrinsic complexity of turn-taking games to predict participants' reaction times. In M. Knauff, M. Pauen, N. Sebanz, and I. Wachsmuth, (Eds.), Proceedings of the 35th annual conference of the Cognitive Science Cociety (pp. 1426-1432). Austin, TX: Cognitive Science Society.

Szymanik, J., \& Verbrugge, R. (Eds.) Proceedings of the Logic and Cognition Workshop at ESSLLI 2012, Opole, Poland, August, 13-17 2012, vol.883 of CEUR Workshop Proceedings. CEUR-WS.org, 2012. http://ceur-ws.org/Vol-883.

Tenenbaum, J. B., Kemp, C., Griffiths, T. L., \& Goodman, N. D. (2011). How to grow a mind: Statistics, structure, and abstraction. Science, 331(6022), 1279-1285.

van Rooij, I. (2008). The tractable cognition thesis. Cognitive Science, 32(6), 939-984. 